\title{
Interaction Region Magnets for VLHC *
}

\author{
Ramesh Gupta and Michael Harrison
}

\begin{abstract}
The interaction region (IR) magnets for the proposed very large hadron collider (VLHC) require high gradient quadrupoles and high field dipoles for high luminosity performance. Moreover, the IR magnets for high energy colliders and storage rings must operate in an environment where the amount of energy deposited on superconducting coils is rather large. In the case of doublet IR optics with flat beams, the design of the first 2-in-1 quadrupole defines the geometry and pole tip field in this and other IR magnets. This paper will present a novel design of this magnet that allows a very small separation between the two apertures. A brief discussion of the conceptual magnetic design of this and other magnets for interaction regions is given. The influence of critical current density in superconductor (a higher value of which is most beneficial to high performance $\mathrm{IR}$ magnet design) is also discussed. Since High Temperature Superconductors (HTS) retain most of their critical current density at high fields and at elevated temperatures, they offer an attractive possibility for the IR magnet designs of future colliders or upgrades of present colliders.
\end{abstract}

Index Terms-Accelerators, Quadrupoles, Interaction Region and High Temperature Superconductors.

\section{INTRODUCTION}

$\mathrm{T}$ HE interaction region lattice [1] and luminosity performance of the proposed VLHC-2 [2] depends significantly on a small number of high field magnets. The IR layout is shown in Fig. 1. The design parameters of various $\mathrm{IR}$ magnets are given in Table I. These values of peak field on the conductor can only be reached by brittle materials such as $\mathrm{HTS}$ and $\mathrm{Nb}_{3} \mathrm{Sn}$. To deal with the large energy deposited by decay particles from the experimental regions, a large margin is allowed in quadrupoles. Developing HTS technology for IR magnets is particularly attractive as it permits a large temperature margin. Given the importance of a few magnets, state of the art superconductors are used in the IR design. The amount of superconductor used in the magnet will go down and the design will become simpler with the further improvements in the superconductor technology.

The following are the major considerations in the design of VLHC-2 IR magnets:

- Small aperture (specially in quadrupoles for generating high gradients)

- Brittle superconductors that must be used for generating high field/gradient

Manuscript received September 24, 2001.

The authors are associated with the Brookhaven National Laboratory, Upton, NY 11973 USA. Email: gupta@bnl.gov and harrison@bnl,gov.

"This work was supported by U.S. Department of Energy under contract number DE-AC02-98CH10886,
- Large Lorentz forces (associated with high fields)

- Small separation between the two apertures (associated with the doublet optics)

The coil ends of conventional cosine $(\theta)$ designs put a practical limit on the minimum aperture, particularly in quadrupole magnets made with brittle material. To overcome this and other limitations, the VLHC-2 IR region is based on non-traditional magnet designs with racetrack coils. The design philosophy adopted here is in part similar to the common coil design [3] used in main dipole magnets. These are conductor friendly designs with large bend radii and are suitable for containing large Lorentz forces.

TABLE I: DESIGN PARAMETERS OF VLHC-2 INTERACTION REGION MAGNETS

\begin{tabular}{ccccccc}
\hline Magnet Field Gradient & Peak Field & Aperture & Length & Type \\
\hline D1A & $16 \mathrm{~T}$ & - & $16.7 \mathrm{~T}$ & $25 \mathrm{~mm}$ & $12.1 \mathrm{~m}$ & $1-$ in-1 \\
D1B & $12 \mathrm{~T}$ & - & $12.5 \mathrm{~T}$ & $50 \mathrm{~mm}$ & $6.0 \mathrm{~m}$ & 1 -in-1 \\
D2 & $12 \mathrm{~T}$ & - & $12.5 \mathrm{~T}$ & $50 \mathrm{~mm}$ & $11.1 \mathrm{~m}$ & 2 -in-1 \\
Q1A & - & $400 \mathrm{~T} / \mathrm{m}$ & $\sim 12 \mathrm{~T}$ & $30 \mathrm{~mm}$ & $12.4 \mathrm{~m}$ & $2-$ in-1 \\
Q1B & - & $600 \mathrm{~T} / \mathrm{m}$ & $\sim 11 \mathrm{~T}$ & $30 \mathrm{~mm}$ & $12.4 \mathrm{~m}$ & $2-$ in-1 \\
Q2A & - & $600 \mathrm{~T} / \mathrm{m}$ & $\sim 11 \mathrm{~T}$ & $30 \mathrm{~mm}$ & $7.9 \mathrm{~m}$ & $2-\mathrm{in}-1$ \\
Q2B & - & $600 \mathrm{~T} / \mathrm{m}$ & $\sim 11 \mathrm{~T}$ & $30 \mathrm{~mm}$ & $7.9 \mathrm{~m}$ & $2-$-in-1 \\
\hline
\end{tabular}

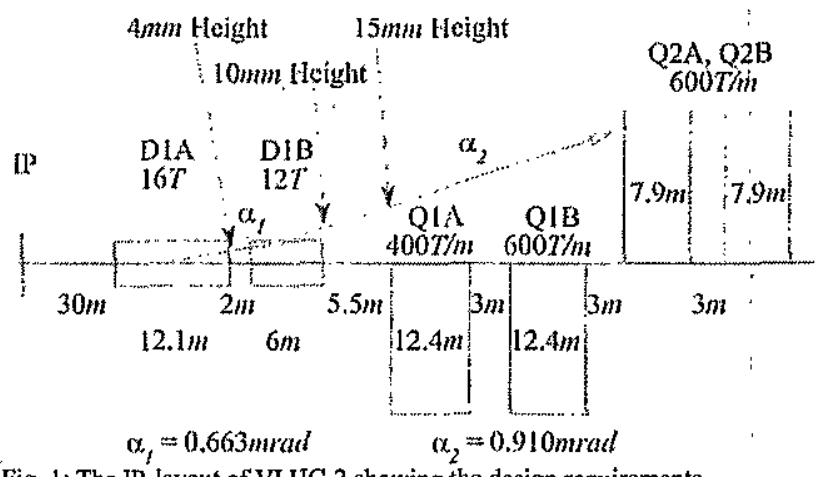

Fig. 1: The IR layout of VLHC-2 showing the design requirements.

\section{IR QUADRUPOLE DESIGN}

In the proposed flat beam optics [1] the minimum separation between the two apertures in Q1A determines the layout of the entire VLHC-2 interaction region and the maximum beam size for the given optics. In addition, it also establishes the maximum pole tip field of this and other magnets. In conventional 2 -in- 1 designs, the minimum separation is determined by the conductor width required for generating field gradient and the support structure required for containing large Lorentz forces. In the proposed design the amount of conductor between the two apertures is much smaller than on any other side and no support structure is required between the two apertures. This brings a large reduction in spacing (by about a factor of five) between the 
two apertures. The cross section of the proposed design is shown in Fig 2. In order to facilitate large bend radii, the return path of all turns is further away from the aperture. Field contours and field lines in the aperture of this magnet are shown in Fig. 3 and $B_{x}$ on vertical axis in Fig. 4. The design is based on "React \& Wind" $\mathrm{Nb}_{3} \mathrm{Sn}$ superconductor with a current density of $2500 \mathrm{~A} / \mathrm{mm}^{2}$ at $12 \mathrm{~T}$. The large bend radii allow the potential use of high temperature superconductors [4]. The magnets made with HTS could operate at elevated temperature arising from the decay particles hitting the coil and/or other parts of the magnet. In addition, one can also remove conductor from the midplane of the magnet, the plane where the decay particles are highly oriented.

Designs based on above philosophy would use much larger amounts of conductor than those would in a conventional quadrupole design. However, the cost of conductor should not be a major factor in designing a few critical high performance IR magnets. The design shown in Fig. 5 allows the neutrals to pass through the space between two apertures. The Lorentz forces are such that a small space can be left in the middle of the internal support structure between the apertures for the passage of neutrals that can be intercepted after Q1A and Q1B.

TABLE Ii: TARGET HARMONICS IN VLHC INERACTION REGION QUADRUPOLES. $<b_{n}>$ AND $<a_{n}>$ ARE THE MEANS OF THE NORMAL AND SKEW TERMS. $d\left(b_{n}\right)$ AND $d\left(a_{n}\right)$ ARE THE UNCERTAINTY IN THE MEAN. $\sigma\left(b_{n}\right)$ AND $\sigma\left(a_{n}\right)$ ARE THE EXPECTED SIGMA. (NOTE: SEXTUPOLE IS $n=2$ ).

\begin{tabular}{ccccccc}
\hline $\mathrm{n}$ & $\left\langle\mathrm{b}_{\mathrm{n}}\right\rangle$ & $\mathrm{d}\left(\mathrm{b}_{\mathrm{n}}\right)$ & $\sigma\left(\mathrm{b}_{\mathrm{n}}\right)$ & $\left\langle\mathrm{a}_{\mathrm{n}}\right\rangle$ & $\mathrm{d}\left(\mathrm{a}_{\mathrm{n}}\right)$ & $\sigma\left(\mathrm{a}_{\mathrm{n}}\right)$ \\
\hline 2 & 0 & 2 & 2 & 3 & 3 & 1 \\
3 & 2 & 2 & 1 & 0 & 1 & 1 \\
4 & 0 & 1 & 1 & 1.5 & 1.5 & 1 \\
5 & 2 & 1 & 0.5 & 0 & 1 & 0.2 \\
6 & 0 & 0.5 & 0.1 & 0.5 & 0.5 & 0.5 \\
7 & 0.5 & 0.1 & 0.1 & 0 & 0.2 & 0.1 \\
8 & 0 & 0.5 & 0.1 & 0.3 & 0.3 & 0.1 \\
9 & 0.5 & 0.1 & 0.1 & 0 & 0.1 & 0.1 \\
\hline
\end{tabular}

The proposed quadrupole design introduces strong coupling and cross talk between the two apertures and the field at the center of the quadrupole is not zero. The superimposition of a dipole field on the quadrupole coils increases the peak field on the conductor and reduces the maximum achievable gradient. The maximum design gradient in Q1A (minimum separation) is, therefore, $400 \mathrm{~T} / \mathrm{m}$ as compared to $600 \mathrm{~T} / \mathrm{m}$ in $\mathrm{Q} 1 \mathrm{~B}, \mathrm{Q} 2 \mathrm{~A}$ and $\mathrm{Q} 2 \mathrm{~B}$ where this effect is much smaller. All four quadrupoles will have a different cross section. The goal is to minimize the cross talk induced harmonics with the exception of the dipole field. By symmetry, the normal even harmonics and skew odd harmonics are theoretically zero. The target field harmonics are given in Table II. These values will be obtained either by design or by correction. Table II includes harmonics from both design and construction errors, however, the harmonic errors are dominated by the design. These harmonics are expected to come down as the design evolves.

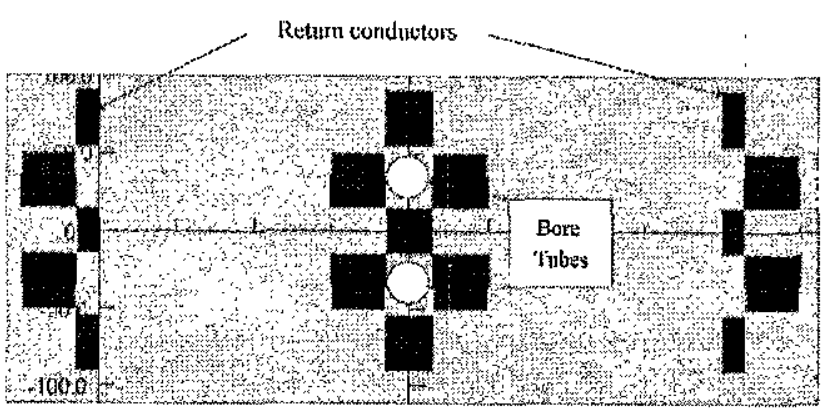

Fig 2: A conceptual design of the proposed 2-in-1 IR Quadrupole Q1A. The design minimizes the spacing between two apertures. Unlike conventional designs, where turns from one octant return to another within the same aperture, in the above design, the conductor returns away from the aperture. The conductor on left side of aperture returns far away on the left side and on the right side of the aperture returns far away on the right side, as shown above. This allows a large bend radius for "React \& Wind" technology.

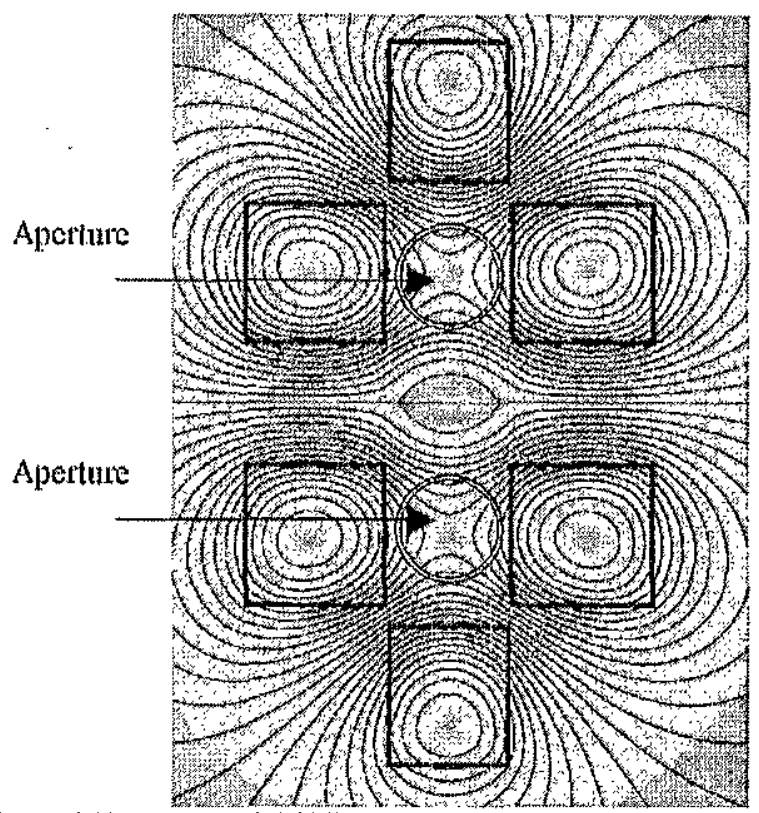

Fig. 3: Field contours and field lines in the aperture region of the proposed design of 2-in-1 quadrupole Q1A. Return conductors are outside the picture.

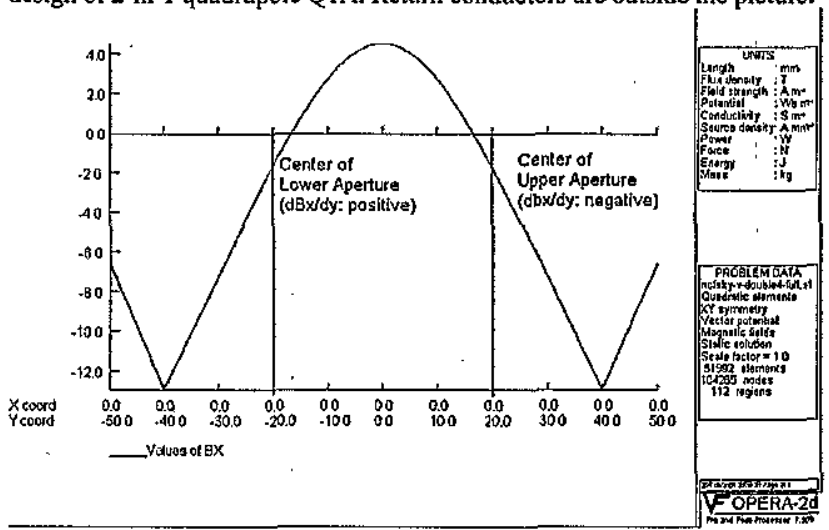

Fig. 4: Horizontal component of the field showing the presence of non-zero field at the center of two apertures. 


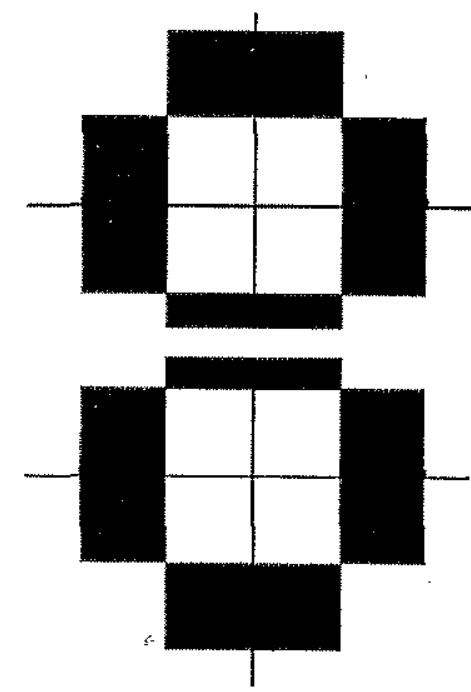

Fig 5: A conceptual design that allows the neutrals from experimental regions to pass through the space between two apertures without hitting the superconducting coils directly. In addition, the space at midplane of two apertures may minimize a direct hit on superconducting coils from the decay particles that are more localized at the midplane.

\section{IR DIPOLES}

The VLHC-2 interaction region uses three types of dipole magnets. The design of all insertion region dipoles is also based on racetrack coils. D1A, DIB (one each on each side) are single aperture dipoles and D2 (two on each side) is a 2in-1 dipole. The operating field in smaller aperture $(25 \mathrm{~mm})$ dipole D1A is $16 \mathrm{~T}$ (Quench field $\sim 18 \mathrm{~T}$ ) and it uses High Temperature Superconductor (BSCCO 2212) in a hybrid design. The operating field in the larger aperture $(50 \mathrm{~mm})$ dipole D1B is reduced to $12 \mathrm{~T}$ to reduce the Lorentz forces associated with larger apertures. This field can be entirely obtained by $\mathrm{Nb}_{3} \mathrm{Sn}$ superconductor.

IR region will also contain a number of corrector magnets. The higher order harmonic correctors could be based on multi-layer coils within the same coldmass, as in RHIC [5].

\section{CONDUCtor Performance AND MAGnet Design}

\section{A. Basic Magnet Design Consideration}

In dipoles and in large aperture and/or low gradient quadrupoles, the required coil thickness $(t)$ to generate a given field or gradient increases linearly. However, the required coil thickness approaches an exponential dependence in small aperture and/or high gradient quadrupoles. This is because the central field $\left(B_{0}\right)$ in cosine $(\theta)$ dipoles is given by

$$
B_{0}=J_{0} * \mu_{0} * t / 2 \text {, }
$$

and the gradient $(\mathrm{G})$ in cosine (20) quadrupole is given by

$$
G=J_{0} * \mu_{0} / 2 * \ln (1+t / a)
$$

where $\mu_{0}$ is the permeability of vacuum and $a$ is the coil radius. This makes the requirement of high operating current density $\left(J_{0}\right)$ at the operating field more critical in high gradient, low aperture quadrupoles, as it remains linear in all cases.

\section{B. Critical Current Density and Magnet Design}

The operating or overall current density $\mathrm{J}_{0}$ depends on the critical current density $J_{\mathrm{sc}}$, the maximum allowed current density in copper $\mathrm{J}_{\mathrm{cu}}$ at quench and insulation and other cable parameters. The maximum acceptable value of $\mathrm{J}_{\mathrm{cu}}$ in modern magnets has been about $1500 \mathrm{~A} / \mathrm{mm}^{2}$. This means that for higher $J_{s c}$, the amount of copper needs to be increased to maintain the maximum value of $\mathrm{J}_{\mathrm{cu}}$. This means that the gain in $J_{o}$ from an increase in $J_{s c}$ above the acceptable copper current density becomes small, as shown in Figs. 6, 7 and 8. Fig. 8, gives the computed coil thickness for obtaining various fields using the type of $\mathrm{J}_{0}\left(\mathrm{~J}_{\mathrm{sc}}, \mathrm{J}_{\mathrm{cu}}\right)$ vs. $B$ fit obtained in Fig. 7.

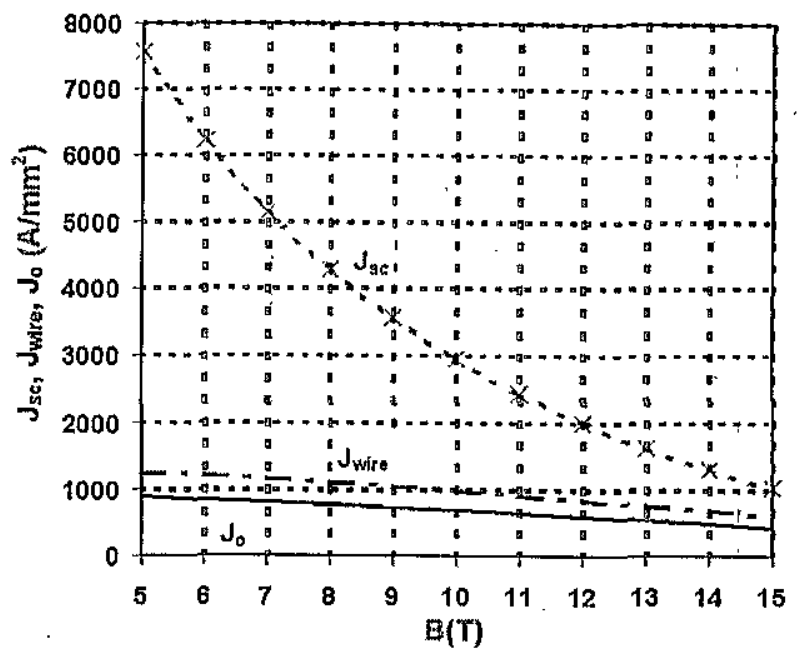

Fig. 6: Critical current density $\left(J_{\mathrm{sc}}\right)$, current density in wire (Jwire) and overall current density $\left(J_{0}\right)$ as a function of field in $\mathrm{Nb}_{3} \mathrm{Sn}$ superconductor for maintaining a copper curtent density $\left(\mathrm{J}_{\mathrm{cu}}\right)$ of $1500 \mathrm{~A} / \mathrm{mm}^{2}$.

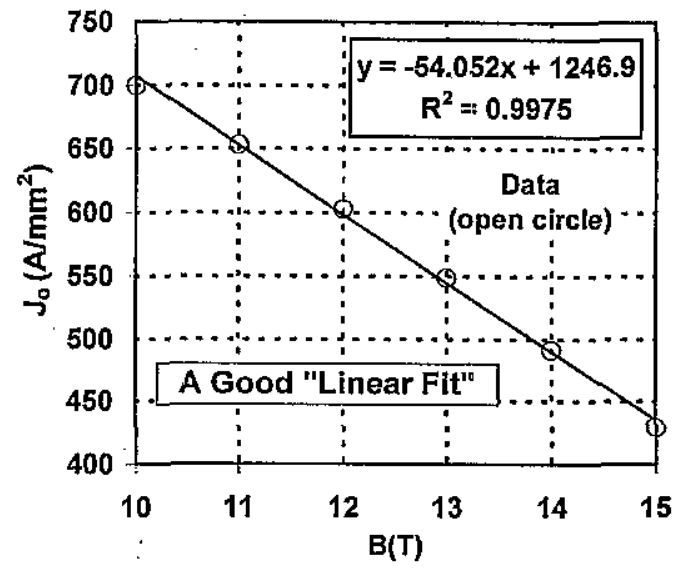

Fig. 7: A linear fit is obtained between $\mathrm{J}_{\mathrm{o}}$ and field. The fit parameters depend on $\mathrm{J}_{\mathrm{sc}}$ and $\mathrm{J}_{\mathrm{cu}}$.

\section{HTS in Accelerator Magnets}

Performance of High Temperature Superconductors (HTS) has been continuously improving [6]. Fig. 9 shows the measured critical current density as of year 2000 in low temperature superconductor (LTS) and in HTS in a wire that can be made in lengths of about 100 meter or longer. The critical current density of HTS becomes more than that of 
LTS at $\sim 13 \mathrm{~T}$ and above. However, the complete benefit of higher critical current density in HTS is not realized in magnet design, as only $1 / 4$ of the present HTS wire is superconductor. The remaining $3 / 4$ is silver that is much larger than required. In HTS wire, the silver plays a similar role as copper does in low temperature superconductors. In high field magnets built with LTS, the superconductor can be kept about $1 / 2$ of the wire volume, depending on the tolerable current density in copper at quench. In Tables III and IV, we compare the designs at various fields where LTS coils are replaced by HTS coils with the following design restrictions. In both HTS and LTS magnets, the current density in copper or silver is $1500 \mathrm{~A} / \mathrm{mm}^{2}$ quench. Moreover, in HTS magnets, $\mathrm{Ag} / \mathrm{SC}$ ration is at least $3: 1$. Table III is based on the critical current density available in year $2000\left(2200 \mathrm{~A} / \mathrm{mm}^{2}\right.$ in $\mathrm{Nb}_{3} \mathrm{Sn}$ and $2000 \mathrm{~A} / \mathrm{mm}^{2}$ in HTS). Table IV is extrapolation for future $\left(3000 \mathrm{~A} / \mathrm{mm}^{2}\right.$ in $\mathrm{Nb}_{3} \mathrm{Sn}$ and $4000 \mathrm{~A} / \mathrm{mm}^{2}$ in HTS). All values are given at $12 \mathrm{~T}$ and $4.2 \mathrm{~K}$ and it is possible that the values extrapolated for future may be realized in $\sim 5$ years.

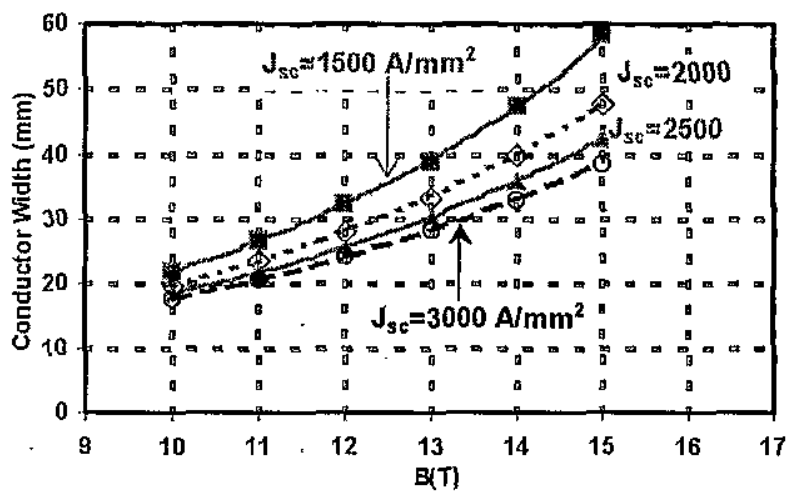

Fig. 8: Coil thickness $(t)$ required to generate various fields for conductor having a critical current density of $1500,2000,2500$ and $3000 \mathrm{~A} / \mathrm{mm}^{2}$ at 12 $\mathrm{T}$ and $4.2 \mathrm{~K}$. Reduction in coil thickness from the improvement in critical current density in superconductor is smaller at lower fiold but significant at higher fields. Jo is obtained using the type of fit shown in Fig. 7.

The IR quadrupole design shown in Fig. 2 is a "conductor friendly" design. It is based on racetrack coils with large bent radius (similar in philosophy to the common coil design [3]) and therefore it allows the use of HTS cables despite their brittle nature. HTS tapes can be bent in smaller radii because of their small thickness as compared to wire and therefore might be attractive in lower aperture magnets. However, in all cases field quality issues, particularly the issues related to persistent current, need to be studied. Their influences on the beam dynamics need to be examined and ways to reduce their magnitude in magnets are being explored.

TABLE 11I: COMPUTED FIELDS IN DIPOLES WHEN THE $\mathrm{Nb}_{3}$ Sn COILS ARE REPLACED BY HTS COILS. THESE DESIGNS ARE BASED ON THE CONDUCTOR PERFORMANCE AS OF YEAR 2000

\begin{tabular}{cc}
\hline $\mathrm{All} \mathrm{Nb}_{3} \mathrm{Sn}$ Coils & All HTS Coils \\
\hline $12 \mathrm{~T}$ & $5 \mathrm{~T}$ \\
$15 \mathrm{~T}$ & $13 \mathrm{~T}$ \\
$18 \mathrm{~T}$ & $19 \mathrm{~T}$ \\
\hline
\end{tabular}

TABLE IV: COMPUTED FIELDS IN DIPOLES WHEN THE Nb3Sn COILS ARE REPLACED BY HTS COILS. THESE DESIGNS ARE BASED ON THE EXPECTED CONDUCTOR PERFORMANCE IN NEAR FUTURE $(\sim 5$ YEARS).

\begin{tabular}{cc}
\hline All $\mathrm{Nb}_{3} \mathrm{Sn}$ Coils & All HTS Coils \\
\hline $12 \mathrm{~T}$ & $11 \mathrm{~T}$ \\
$15 \mathrm{~T}$ & $16 \mathrm{~T}$ \\
$18 \mathrm{~T}$ & $22 \mathrm{~T}$ \\
\hline
\end{tabular}

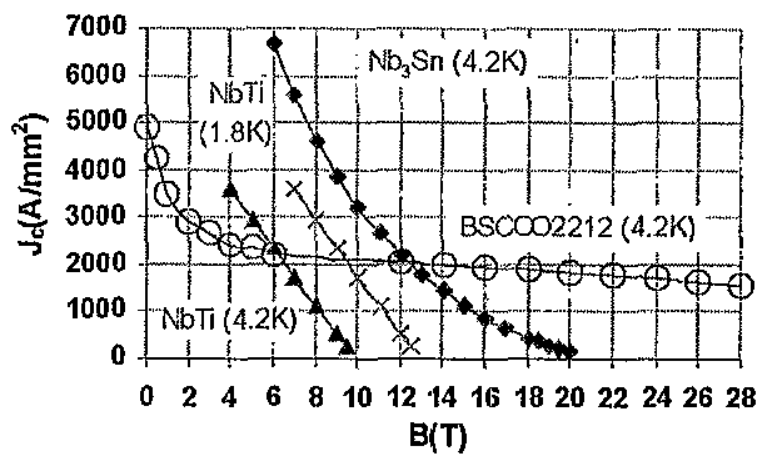

FIG. 9: CRITICAL CURRENT DENSTTY AS A FUNCTION OF FIELD IN YEAR 2000.

\section{HTS COIL AND MAGNET R\&D PROGRAM AT BNL}

The superconducting magnet division at BNL has started a challenging HTS magnet $R \& D$ program. The program is discussed in detail elsewhere [4]. The use of HTS is particularly attractive in IR magnets because they maintain a large critical current density at very high field and can operate at elevated temperatures (caused by decay particles hitting the coil and/or nearby structure) without much loss in critical current density. Moreover, given the present high cost of HTS, it is likely that HTS-based accelerator magnets will find their first use in high performance interaction regions. This is because of the fact that in the case of IR's a few magnets have a major impact on the luminosity performance of the machine. Therefore, in this case, the performance rather than the material cost should be the prime motivation for developing new magnet designs and technologies.

\section{ACKNOWLEDGEMENTS}

We appreciate discussions with $M$. Anerella, W. Fischer, B. Parker, S. Peggs, F. Pilat, S. Tepekian, D. Trbojevic, P. Wanderer and E. Willen. We thank P. Wanderer for his constructive comments on the manuscript of this paper.

\section{REFERENCES}

[1] VLHC Accelerator Physics, unpublished, internal reports BNL CAD/AP/49 and FNAL TM-2158 June 29, 2001.

[2] Design Study for a Staged VLHC, unpublished, Fermilab Internal Report TM-2179, June 4, 2001.

[3] R. Gupta, "A Common Coil Design for High Field 2-in-1 Accelerator Magnets," Proceedings of the 1997 Particle Accelerator Conference.

[4] R, Gupta, et al, "R\&D for Accelerator Magnets with React \& .Wind High Temperature Superconductors," this conference.

[5] A. Morgillo, et al., "Superconducting $8 \mathrm{~cm}$ Corrector Magnets for the Relativistic Heavy Ion Collider (RHIC)," Proceedings of the 1995 Particle Accelerator Conference.

[6] T. Hascgawa, "HTS Conductors for Magnets," this conference. 